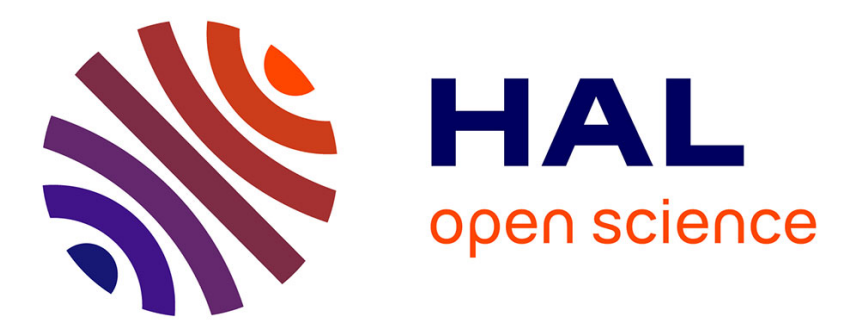

\title{
Polyamorphic transition of germanium under pressure
} Emiliano Principi, Andrea Di Cicco, Frédéric Decremps, Alain Polian, Simone de Panfilis, Adriano Filipponi

\section{To cite this version:}

Emiliano Principi, Andrea Di Cicco, Frédéric Decremps, Alain Polian, Simone de Panfilis, et al.. Polyamorphic transition of germanium under pressure. Physical Review B: Condensed Matter and Materials Physics (1998-2015), 2004, 69 (20), pp.e555 - e559. 10.1103/PhysRevB.69.201201 . hal01921237

\section{HAL Id: hal-01921237 https://hal.sorbonne-universite.fr/hal-01921237}

Submitted on 13 Nov 2018

HAL is a multi-disciplinary open access archive for the deposit and dissemination of scientific research documents, whether they are published or not. The documents may come from teaching and research institutions in France or abroad, or from public or private research centers.
L'archive ouverte pluridisciplinaire HAL, est destinée au dépôt et à la diffusion de documents scientifiques de niveau recherche, publiés ou non, émanant des établissements d'enseignement et de recherche français ou étrangers, des laboratoires publics ou privés. 


\title{
Polyamorphic transition of germanium under pressure
}

\author{
Emiliano Principi, Andrea Di Cicco* \\ INFM, *INFN-LNF, Università di Camerino, Dip. di Fisica, \\ via Madonna delle Carceri, 62032 Camerino (MC), Italy \\ F. Decremps, A. Polian \\ Physique des Milieux Condensés, CNRS, \\ UMR 7602, Université Pierre et Marie Curie B 77, \\ 4 place Jussieu, F-75252 Paris CEDEX 05, France \\ S. De Panfilis \\ ESRF, B.P. 220 38043 Grenoble Cedex, France \\ A. Filipponi \\ INFM, Università dell'Aquila, Dip. di Fisica, \\ via Vetoio 67010 Coppito, L'Aquila, Italy
}

(Dated: January 7, 2004)

\begin{abstract}
Pressure-induced transformations in the atomic and electronic structure of amorphous germanium (a-Ge) have been investigated by using x-ray absorption spectroscopy (XAS) combined with energy-scanning x-ray diffraction. Our data show that an abrupt change in the local structure and in the electron states near the Fermi level occurs in evaporated a-Ge at a pressure of about $8 \mathrm{GPa}$. The transformation is clearly detectable by a change in the shape and energy shift in the near-edge structures and by an increase of the average first-neighbor distance measured by XAS. The occurrence of this polyamorphic transition is discussed in light of the recent advances in the study of multiple dense fluid phases.

PACS numbers: $64.70 .-\mathrm{p}, 61.10 . \mathrm{Ht}, 61.43 . \mathrm{Dq}, 61.50 . \mathrm{Ks}$
\end{abstract}

*Electronic address: emiliano.principi@unicam.it; URL: http://gnxas.unicam.it/〜principi 
Recent studies have revealed a complex behavior in a number of disordered systems that point to the occurrence of multiple dense fluid phases, distinguished by changes in thermodynamic quantities and by their microscopic structure, for same chemical composition. [1-4] These results have obvious important implications for the development of liquid and glass science. In particular, the possibility of producing different glassy structures for a given substance[4-6] can have remarkable consequences both in basic and applied science, in view of the almost ubiquitous presence of glasses in nature and technology.

However, the study of polymorphic transitions in disordered matter usually requires the exploration of a wide temperature and pressure range in order to observe detectable variations in physical quantities associated with a liquid or glassy system. The transition is usually characterized by changes in energy, entropy and density much smaller than those for the usual liquid-gas transition, as two fluid phases of similar density are involved. The coexistence line could lie well below the crystal-liquid transition, thus the existence of distinct fluid phases can be obscured by crystal nucleation but could be revealed in the undercooled liquid or by changes of the amorphous solid (polyamorphism).

Natural candidates for possible observation of fluid-fluid phase transitions include all disordered systems showing a local open molecular structure at low pressure which can be forced to increase both density and local atomic coordination by applying an external pressure. A broad range of simple substances belongs to this class of systems, and in particular water, Si, Ge, C and their oxides form liquid and glass materials where the local tetrahedral coordination plays a prominent role. Evidence for polyamorphism in water has been obtained from computer simulations and thermodynamic data[5]. There are also reliable experimental data pointing to the presence of multiple disordered phases in the so-called "strong" glass-formers $\mathrm{SiO}_{2}$ and $\mathrm{GeO}_{2} \cdot[7,8]$ Recent works using x-ray diffraction (XRD) at high pressure have shown that liquid phases with different local structures are obtained by application of pressure for systems with tetrahedral coordination characterized by complex solid phase diagrams $[9,10]$.

The present work is aimed to shed new light on the polyamorphic nature of the simplest glassy system with local tetrahedral structure: amorphous germanium (a-Ge). To achieve this aim, we have used the x-ray absorption spectroscopy (XAS) whose exceptional sensitivity to the local structure makes us able to detect and study in details tiny structural transformations. Germanium, and silicon of course, can be considered archetypal systems for 
understanding the relationship between polymorphism and interactions at the atomic scale. There is a competition of bonding mechanisms reflected naturally in the phase diagram of those systems, which are simple low-density tetrahedral semiconductors at ambient pressure and temperature but are metallic and denser at high temperature in the liquid state or under high pressure conditions in the crystalline state (see for example 11 and ref. therein). Germanium can be easily undercooled up to $250 \mathrm{~K}$ below the melting point, but the system crystallizes "catastrophically" [12] and tetrahedral a-Ge can be only produced by different routes (evaporation on a cold substrate for example). A two-phase liquid model has been proposed $[6,13]$ to understand this behavior and the observation of metalization of thin a-Ge films $[14,15]$ under hydrostatic pressure above $6 \mathrm{GPa}$. On the other hand, no modifications of the structure of a-Ge were observed for pressures below $10 \mathrm{GPa}$ using XAS,[16, 17] probably for the limited resolution of the experiments and for limitations in the data-analysis techniques. More recently, x-ray diffraction and Raman experiments showed evidence of a transition from a low density to a high density disordered phase in amorphous Si.[18] Theoretical calculations have indicated that a-Si and a-Ge undergo transitions to denser structures although transition pressures differ from those observed experimentally.[19, 20]

In this work, we present new a-Ge XAS measurements performed at the ESRF, using the advanced set up available at the BM29 beamline [21,22] which allows the simultaneous collection of low-noise and high resolution XAS data and energy-scanning XRD patterns used to monitor pressure and crystallization of the sample. Samples of a-Ge have been prepared by evaporation of high-purity Ge under high vacuum conditions $\left(10^{-5}\right.$ mbar $)$ onto Kapton films. The absence of Bragg peaks associated with Ge crystalline phases was confirmed by the powerful seven-channel collimator system available at BM29[21]. Absence of sample oxidation was also confirmed by near-edge XAS spectra. The a-Ge sample used for combined XAS and XRD measurements under pressure was placed in a $2 \mathrm{~mm}$ graphite hollow cylinder along with a mixture of compacted $\mathrm{BN}$ and $\mathrm{LiF}$ powders used as pressure markers, and inserted in a $7 \mathrm{~mm}$ boron-epoxy gasket conceived for the "Paris-Edinburgh" large-volume press.[21, 23]

High quality a-Ge XAS spectra (Ge $K$-edge) and related diffraction patterns have been collected up to $8.3 \mathrm{GPa}$ at room temperature. The highest pressure was reached with an external oil pressure of about 1200 bars and a minimal gap of $0.15 \mathrm{~mm}$ between the WC anvils for the collection of the x-ray measurements. The experimental XAS data have been 
analyzed using state-of-the-art data-analysis techniques (GNXAS package [24, 25]).

Raw XAS data of a-Ge measured cycling the external pressure are presented in Fig. 1. An irreversible change of the shape of the spectrum is clearly revealed by the measured XAS spectra reported in the upper panel of Fig. 1. The modification is obtained at a pressure $P_{c} \sim 8 \mathrm{GPa}$ and no germanium Bragg peaks are detected throughout the entire set of measurements. A clear shift of the absorption edge toward lower energies is observed in a-Ge at high pressures. The position of the absorption edge has been measured with an accuracy better than $0.1 \mathrm{eV}$ using a c-Ge reference sample (see Fig. 1 of ref. 22) measured simultaneously with a third ionization chamber. The energy shift, similarly to the case of metallic liquid Ge, $[12,26]$ is associated with the presence of electron states inside the gap. In liquid Ge this is clearly due to the metalization, while in the present case this could be associated with an increased structural disorder corresponding to the creation of both localized and delocalized states inside the gap. The energy shift from the position of the edge measured in crystalline germanium (c-Ge I, diamond structure) is about $0.5 \mathrm{eV}$ for modified a-Ge (a-Ge II in Fig. 1), much greater of that observed for the "as-deposited" a-Ge one (about $0.2 \mathrm{eV}$ ).

In Fig. 2 we report the a-Ge XAS structural signal $k \chi(k)$, obtained by suitable normalization of the original data, for increasing pressure. High-quality experimental data at high pressure are shown with superimposed best-fit XAS simulations (dashed lines). The a-Ge $k \chi(k)$ signal is a regular oscillation as a function of the photoelectron wave-vector $k=\sqrt{2 m\left(E-E_{e}\right)} / \hbar\left(E_{e}\right.$ being the threshold energy) and it is associated practically only with the first-neighbor Ge-Ge distance distribution, as shown by the leading Fourier Transform (FT) peak located just above $2 \AA$ (see upper panel of Fig. 2). The gradual shortening of the first-neighbor average distance, and its sudden expansion above $P_{c} \sim 8 \mathrm{GPa}$ is revealed by the changes of the $k \chi(k)$ leading frequency (arrows) and is clearly visible in the Fourier Transform. While the shortening of the distance is a natural consequence of the application of the external pressure, the sudden expansion reveals that a different modification of the local structure takes place at about $8 \mathrm{GPa}$.

This transformation is irreversible, as shown in Fig. 3, where the normalized x-ray absorption coefficient at constant energy $\left(E_{a}=11102.6 \mathrm{eV}\right.$, see inset in Fig. 1) and the maximum height of the first FT peak are reported. A discontinuous change of more than 5 $\%$ of the x-ray absorption is observed at $P_{c}$, while the FT height changes of about $15 \%$ moving 
visibly to longer distances. The transition between two different amorphous structures at $P_{c}$ is in agreement with the results of a two-phase liquid model (shown in Fig. 3, lower panel) for which a denser metallic phase is favored at high pressure. More recent calculations[19] pointing to the presence of an irreversible transition of a-Ge at about $12.5 \mathrm{GPa}$ to a denser phase with a reduced optical gap are in qualitative agreement with present results. The actual transition pressure can obviously depend on the sample preparation procedure and on the accuracy of the calculations but the existence of different amorphous phases with distinct structural and electronic properties is unambiguously assessed in the present experiment.

A detailed analysis of the XAS signals presented in Fig. 2 reveals important details of the structural change occurring at $P_{c}$. XAS data collected during the pressure cycle were analyzed using a simple first-neighbor model within the GNXAS package (see ref. 12, 24, 25). The agreement between experimental data and simulations is excellent as shown in Fig. 2 . The first-neighbor distribution has been modeled as a $\Gamma$-like function[12] which depends on a limited number of structural parameters: coordination number $N$, average distance $R$, bondlength variance $\sigma^{2}$, and skewness $\beta$. The skewness $\beta$ was found practically negligible for the whole set of XAS data $(\beta \sim 0.2)$ so the final first-neighbor distributions can be considered Gaussian within the typical error limits. The average first-neighbor distance $R$ is the most accurate structural quantity obtained by this XAS experiment (typical error is below $0.005 \AA$ ). The trend of $R$ as a function of pressure is reported in Fig. 4, upper panel. Different regimes can be identified for increasing pressures (upstroke): i) rapid shortening of the average distance for moderate pressures $(\mathrm{P}<1 \mathrm{GPa})$; ii) an intermediate region $(\mathrm{P}$ $<6 \mathrm{GPa})$ where the local structure is much less compressible; iii) a "plateau" region $(\mathrm{P}<8$ $\mathrm{GPa}$ ) where the average distance is practically constant; iv) an increase of $R$ of about 0.025 $\AA$ above the pressure $P_{c} \sim 8 \mathrm{GPa}$. The smooth compression of c-Ge I (diamond) is shown for comparison as a dotted line in Fig. 4 (see 11 and ref. therein). The trend of the average distance upon decompression is quite regular and the expansion is found to be similar to that of the metastable crystalline structure ST12 ( $\mathrm{P} 4_{3} 2_{1} 2$ Ge III), a denser crystalline phase with local tetrahedral units (dotted curve refers to the $\mathrm{ST} 12$ structure at about $100^{\circ} \mathrm{C}[11]$ ). The average bondlength at ambient pressure is found much longer than that of the initial a-Ge structure confirming the irreversibility of the process.

The trend of the bondlength variance $\sigma^{2}$ is also particularly interesting. Looking at Fig. 4, lower panel, we observe that the variance decreases drastically for increasing pressures (lower 
than $2 \mathrm{GPa}$ ), and then remains constant within the uncertainty below $P_{c}$. The transition to the new amorphous structure is accompanied by a substantial increase of the variance (about $35 \%$ ). The larger disorder of the structure is retained down to ambient pressure. At the transition, the best-fit effective coordination number is found to increase of about $12 \%(N \sim 4.5)$. As it is well-known, there is a large numerical correlation between best-fit $N$ and $\sigma^{2}$ obtained by XAS data, as they both affect the $\chi(k)$ amplitude. For this reason, the uncertainty on both parameters is quite large (about 10\%). However, the increase in coordination number is perfectly reasonable taking into account the density increase at high pressures and the expansion of the bondlength above the transition pressure.

In conclusion, our data show that evaporated a-Ge transforms under high-pressure to a new a-Ge form whose structural and electronic states properties are significantly different. This well-defined transition occurs at $8 \mathrm{GPa}$ and is characterized by an increase of the average first-neighbor distance accompanied by a larger distance spread and increase of the average coordination number. The transition is found to be irreversible and the new structure is found to show signatures of a metallic character in agreement with theoretical models based on the competition between covalent and metallic bonding. 
[1] P. H. Poole, T. Grande, C. A. Angell, and P. F. McMillan, Science 275, 322 (1997).

[2] C. A. Angell, K. L. Ngai, G. B. McKenna, P. F. McMillan, and S. W. Martin, Journal of Applied Physics 88, 3113 (2000).

[3] G. Franzese, G. Malescio, A. Skibinsky, S. V. Buldyrev, and H. E. Stanley, Nature 409, 692 (2001).

[4] P. G. Debenedetti and F. H. Stillinger, Nature 410, 259 (2001).

[5] O. Mishima and H. E. Stanley, Nature 396, 329 (1998).

[6] E. G. Ponyatovsky and O. I. Barkalov, Mat. Sci. Rep. 8, 147 (1992).

[7] M. Grimsditch, Phys. Rev. Lett. 52, 2379 (1984).

[8] K. H. Smith, E. Shero, A. Chizmeshya, and G. H. Wolf, J. Chem. Phys. 102, 6851 (1984).

[9] Y. Katayama, T. Mizutani, W. Utsumi, O. Shimomura, M. Yamakata, and K. Funakoshi, Nature 403, 170 (2000).

[10] W. Crichton, M. Mezouar, T. Grande, S. Stolen, and A. Grzechnik, Nature 414, 622 (2001).

[11] A. Di Cicco, A. C. Frasini, M. Minicucci, E. Principi, J. P. Itié, and P. Munsch, Phys. Stat. Sol. (b) $\mathbf{2 4 0}$ (2003).

[12] A. Filipponi and A. Di Cicco, Phys. Rev. B 51, 12322 (1995).

[13] L. I. Aptekar, Sov. Phys. Dokl. 24, 993 (1979).

[14] O. Shimomura, S. Minomura, N. Sakai, K. Asaumi, K. Tamura, J. Fukushima, and H. Endo, Phil. Mag. 29, 547 (1974).

[15] K. Tanaka, Phys. Rev. B 43, 4302 (1991).

[16] J. Freund, R. Ingalls, and E. D. Crozier, J. Phys. Chem. 94, 1087 (1990).

[17] J. P. Itié, A. Polian, D. Martinez-Garcia, V. Briois, A. Di Cicco, A. Filipponi, and A. San Miguel, J. Physique IV 7, C2 (1997).

[18] S. K. Deb, M. Wilding, M. Somayazulu, and P. F. McMillan, Nature 414, 528 (2001).

[19] M. Durandurdu and D. A. Drabold, Phys. Rev. B 66, 41201 (2002).

[20] M. Durandurdu and D. A. Drabold, Phys. Rev. B 67, 212101 (2003).

[21] A. Filipponi, V. M. Giordano, S. D. Panfilis, A. Di Cicco, E. Principi, A. Trapananti, M. Borowski, and J.-P. Itiè, Rev. Sci. Instrum. 74, 2654 (2003).

[22] A. Filipponi, M. Borowski, D. T. Bowron, S. Ansell, A. Di Cicco, S. De Panfilis, and J.-P. 
Itiè, Rev. Sci. Instr. 71, 2422 (2000).

[23] J. M. Besson, R. J. Nelmes, G. Hamel, J. S. Loveday, G. Weill, and S. Hull, Physica B 180 \& 181, 907 (1992).

[24] A. Filipponi, A. Di Cicco, and C. R. Natoli, Phys. Rev. B 52, 15122 (1995).

[25] A. Filipponi and A. Di Cicco, Phys. Rev. B 52, 15135 (1995).

[26] A. Filipponi, M. Borowski, P. W. Loeffen, S. De Panfilis, A. Di Cicco, F. Sperandini, M. Minicucci, and M. Giorgetti, J. Phys.: Condens. Matter 10, 235 (1998). 
FIG. 1: Upper panel: XAS experimental data of a-Ge measured cycling the external pressure. An irreversible change of the shape of the spectrum is observed above a pressure of about 8 GPa. Lower panel: near-edge Ge $K$-edge XAS data of a-Ge as deposited, at high pressure and after the entire pressure cycle, compared with that of c-Ge.

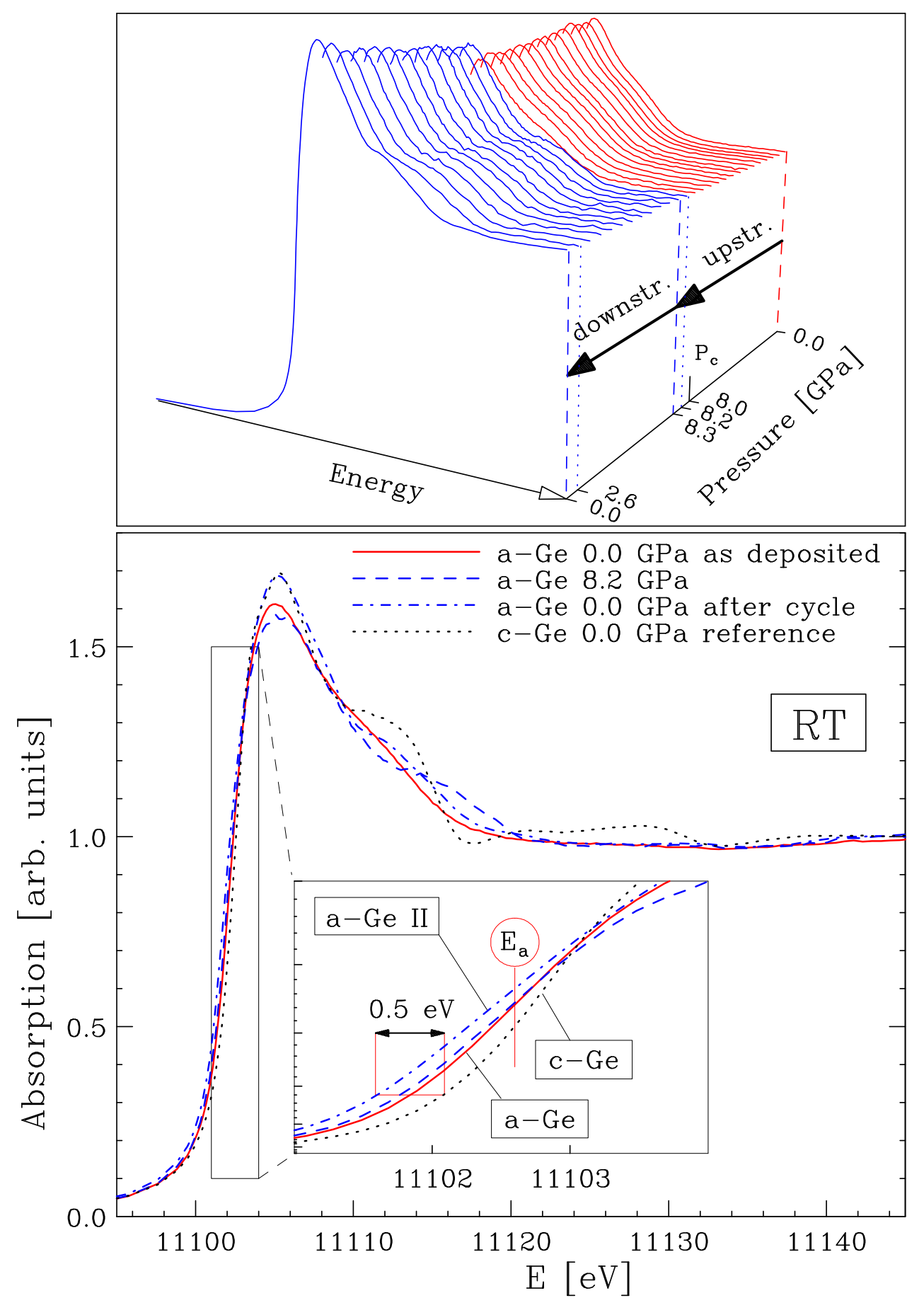


FIG. 2: Experimental (solid) and best-fit (dashed) XAS structural signals $k \chi(k)$ of a-Ge measured for increasing pressure. The gradual shortening of the average distance and its sudden expansion above a pressure of about $8 \mathrm{GPa}$ is indicated by the modification of the $k \chi(k)$ leading frequency (see arrows) and is visible in the Fourier Transform (upper panel and inset).

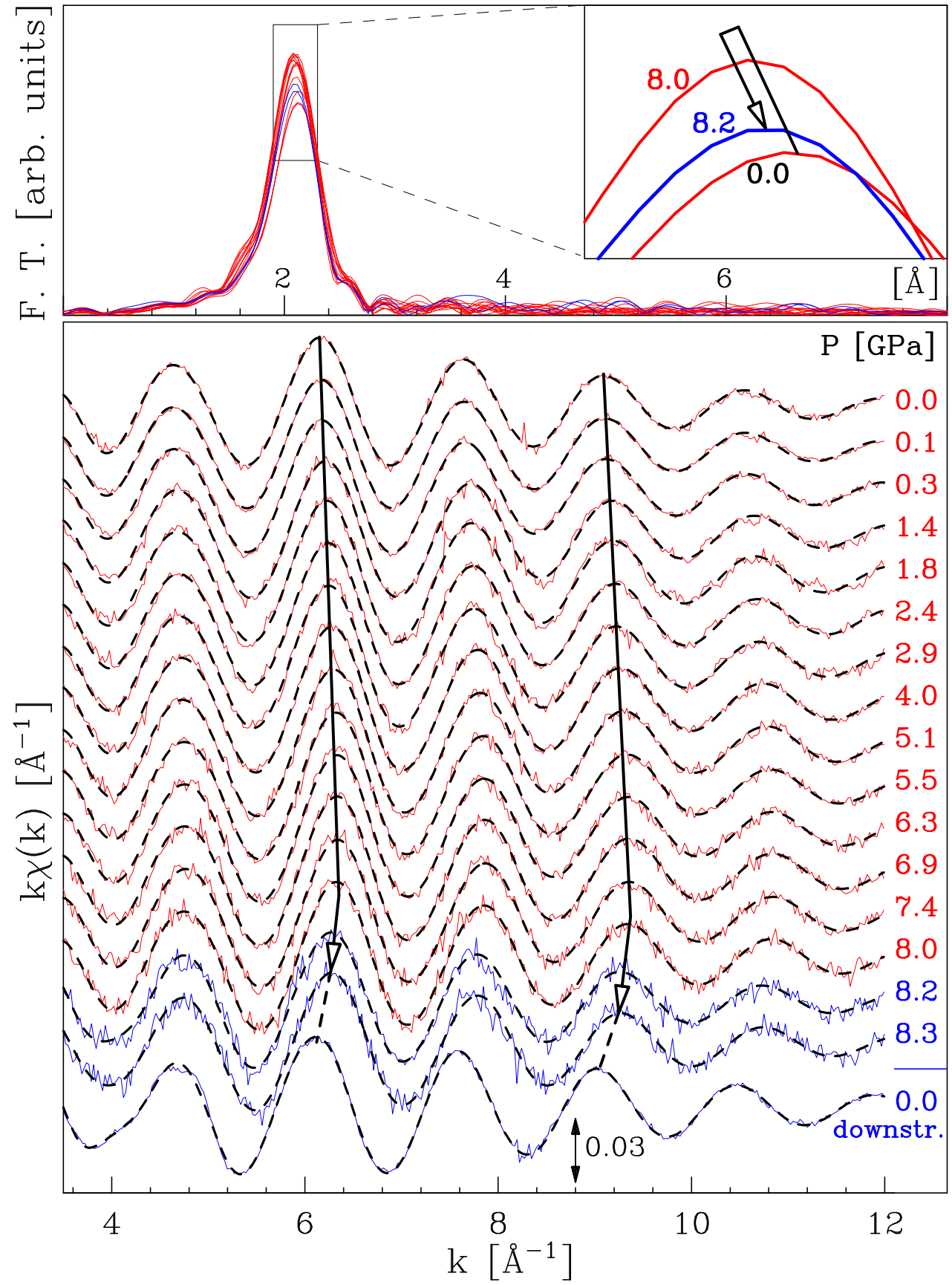


FIG. 3: Upper panel: normalized x-ray absorption at $11102.6 \mathrm{eV}$ photon energy (near the Ge $K$-edge) which clearly shows the irreversible transition to a-Ge II around $8 \mathrm{GPa}$. Open symbols (circles and squares) are used for data obtained increasing the pressure (upstroke). Full symbols refer to data collected upon pressure release (downstroke). Central panel: maximum height of the first peak of the Fourier Transform of the XAS structural signal during the pressure cycle. Lower panel: regions where metallic or covalent bonding are favored in liquid Ge[13] as compared with present results on a-Ge. The hypothetical liquid-liquid coexistence line (thick) and metastability region (cross-hatched) separating a low-density covalent and a higher density metallic liquid are shown on the Ge phase diagram as predicted in ref.13.

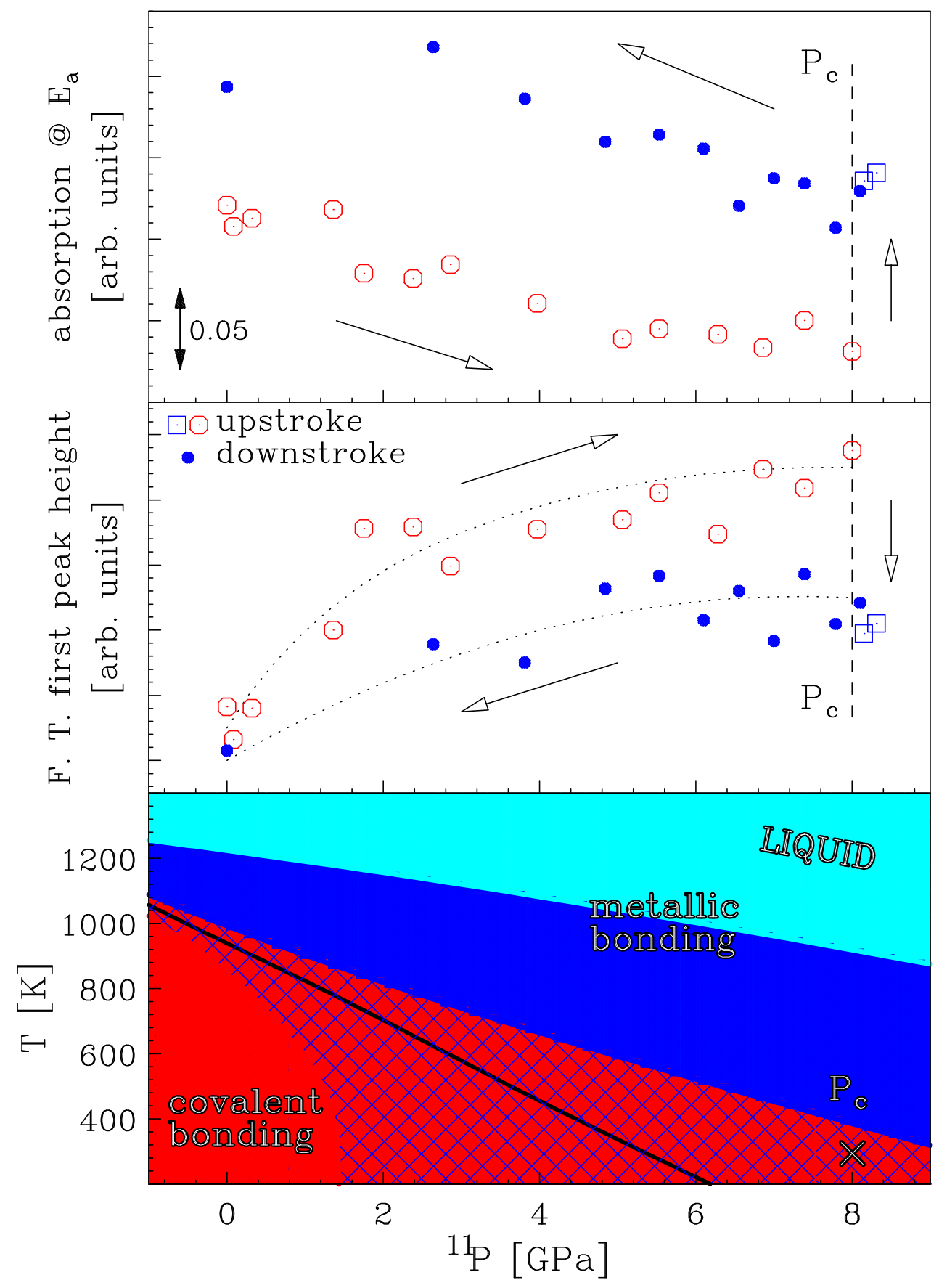


FIG. 4: Average distance (upper panel) and bond variance (lower panel) obtained by XAS dataanalysis of a-Ge at high pressures. Open and full symbols refer to data data obtained increasing (upstroke) and releasing (downstroke) the pressure respectively. Bond distances are compared with the corresponding first-neighbor distances of crystalline Ge obtained by suitable scaling of diffraction data.[11] A significant increase of the average distance and an increase of the disorder (bond variance) and coordination is obtained above $8 \mathrm{GPa}$.

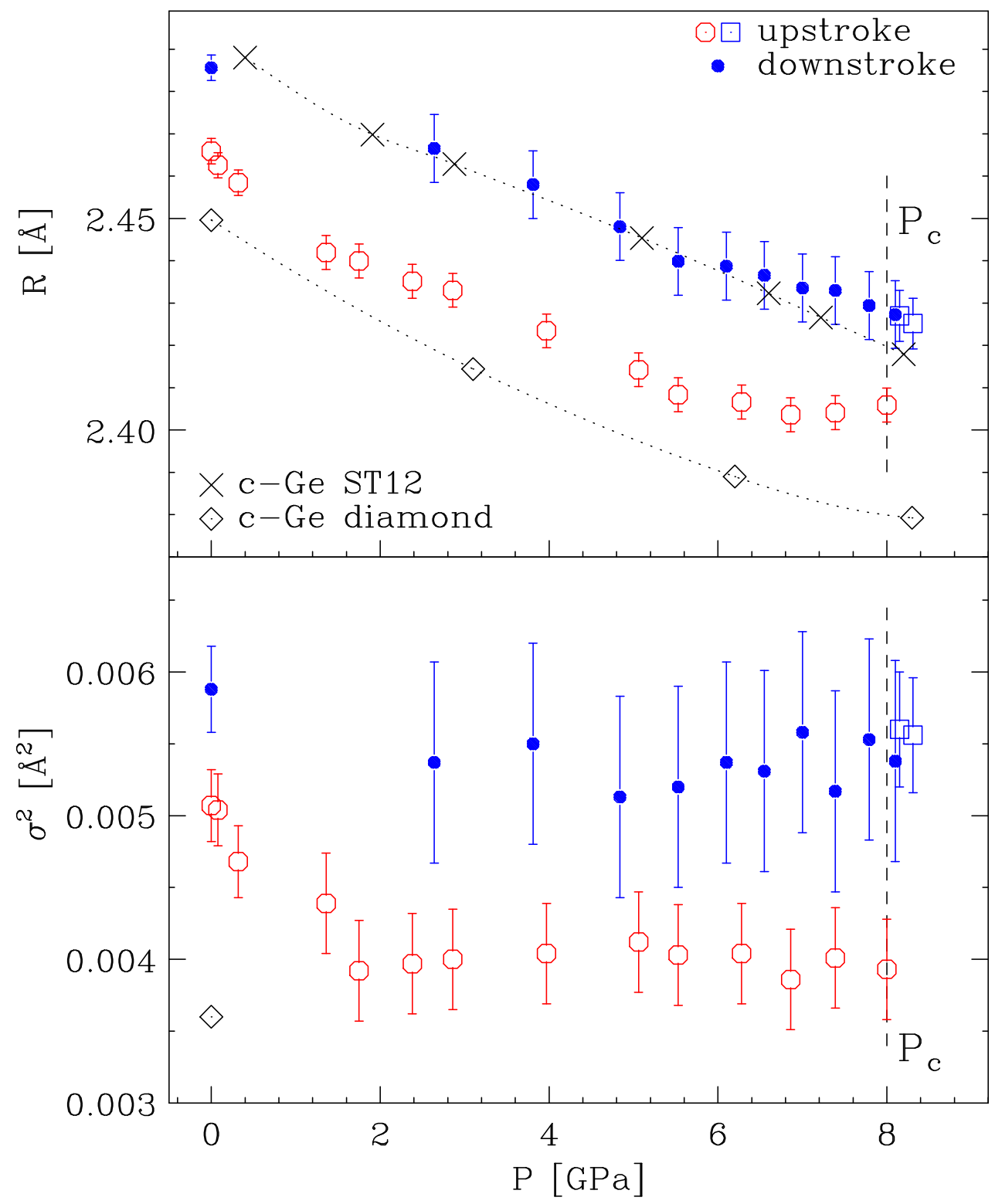

